\title{
Accelerating numerical wave propagation by wavefield adapted meshes, Part II: Full-waveform inversion
}

\author{
Solvi Thrastarson ${ }^{1}$, Martin van Driel ${ }^{1}$, Lion Krischer ${ }^{1}$, Christian Boehm ${ }^{1}$, \\ Michael Afanasiev $^{1}$, Dirk-Philip van Herwaarden ${ }^{1}$, Andreas Fichtner ${ }^{1}$ \\ ${ }^{1}$ Department of Earth Sciences, Institute of Geophysics, ETH Zürich, Sonneggstrasse 5, 8092 Zürich, Switzerland
}

22 August 2019

\begin{abstract}
SUMMARY
We present a novel full-waveform inversion approach which can reduce the computational cost by up to an order of magnitude compared to conventional approaches, provided that variations in medium properties are sufficiently smooth. Our method is based on the usage of wavefieldadapted meshes which accelerate the forward and adjoint wavefield simulations. By adapting the mesh to the expected complexity and smoothness of the wavefield, the number of elements needed to discretise the wave equation can be greatly reduced. This leads to spectral-element meshes which are optimally tailored to source locations and medium complexity. We demonstrate a workflow which opens up the possibility to use these meshes in full-waveform inversion and show the computational advantages of the approach. We provide examples in 2-D and 3-D to illustrate the concept, describe how the new workflow deviates from the standard full-waveform inversion workflow, and explain the additional steps in detail.
\end{abstract}

Pre-print submitted to GJI July 2019

\section{INTRODUCTION}

Since the first applications of seismic tomography (e.g., Dziewoński et al. 1977; Aki et al. 1977) the field has advanced through an increase in quantity and quality of data, which in turn has motivated methodological advancements. Today it is technically possible to utilize the full information from waveforms recorded at seismic stations distributed around the globe in a broad frequency range. To exploit this information, the physical equations describing how a wavefield propagates away from a source and through a potentially complex medium need to be solved accurately.

Relying on numerical wavefield simulations, full-waveform inversion (FWI) is a method to extract information from waveforms, which has become practical comparatively recently, despite its much earlier conceptualisation (Bamberger et al. 1982; Lailly \& Bednar 1983; Tarantola 1984, 1988). Meanwhile, FWI has been applied in global seismology (e.g., French \& Romanowicz 2014; Bozda $\breve{g}$ et al. 2016; Fichtner et al. 2018), in studies of regional Earth structure (e.g., Chen et al. 2007b; Tape et al. 2009; Fichtner et al. 2009; Zhu et al. 2012; Simute et al. 2016; Krischer et al. 2018), at the exploration scale (e.g., Igel et al. 1996; Virieux \& Operto 2009; Sirgue et al. 2010; Warner et al. 2013), and in medical ultrasound tomography (Pratt et al. 2007; Boehm et al. 2018).

FWI requires computation of synthetic waveforms which are compared to observed data. Starting from an initial model (medium), FWI, in a tomographic application, iteratively minimizes waveform misfits by modifying the model in each iteration. The modifications applied to the model are computed using the gradient of the misfit with respect to the model parameters. The gradient is computed either using the adjoint method (e.g., Tarantola 1988; Tromp et al. 2005; Fichtner et al. 2006; Plessix 2006) or the scattering-integral method (e.g., Chen et al. 2007b,a). Both methods compute a gradient with one additional wavefield simulation.

\subsection{Waveform Modelling}

Simulating wave propagation through arbitrary heterogeneous media can only be done numerically. This is a computationally challenging task which has been studied to great extent in recent decades. The spectral-element method (SEM) (Patera 1984; Seriani \& Priolo 1994; Faccioli et al. 1996, 1997) has become a standard method for numerical wave propagation simulations in the community of passive seismology. Several wave propagation solvers based on SEM currently exist (e.g. Komatitsch \& Tromp 2002; Gokhberg \& Fichtner 2016; Cupillard et al. 2012; Afanasiev et al. 2019). These solvers have the capability to accurately model seismic wave propagation through complex 3-D media. The simulations swiftly become computationally expensive, either through an increase in modelled frequency or spatial extent of the model. In an $N$-dimensional medium, the computational cost of the simulation scales with frequency to the power of $N+1$. The $N$ is due to increase in the number of elements needed to discretise the medium in each dimension and the +1 results from the reduced timestep needed to meet the Courant-Friedrichs-Lewy (CFL) criterion (Courant et al. 1928) in explicit time stepping schemes.

Computational cost of FWI scales with simulation cost, the number of sources used in the inversion and the amount of iterations performed. In an ideal case it would be beneficial to include as much data as possible (many sources) and iterate to convergence. It is thus of great importance to reduce the cost of the wave propagation simulations, ideally while still resolving the relevant waveforms. 


\section{Thrastarson et al.}

\subsection{Reducing the computational cost}

Improvements in hardware and algorithm design have contributed to the reduction of the computational burden carried by wave propagation simulations. Moving the SEM to a GPU architecture can result in significant speedups depending on the setup (Komatitsch et al. 2010; Rietmann et al. 2012; Gokhberg \& Fichtner 2016). The implementation of local timestepping (Dumbser et al. 2007; Rietmann et al. 2015) can result in similar speedups for specific problems where a relatively low number of small elements limit the global timestep of the simulation.

Simulation cost has also been reduced with simplifications of the full 3-D problem. AxiSEM, for instance, exploits the approximate axial symmetry of global wavefields by using 2-D meridian slice computations and analytically extending to the full globe (NissenMeyer et al. 2007, 2008, 2014). However, as the medium complexity increases, both in terms of shape and interior structure, the requisite assumptions on symmetry begin to break down.

As a remedy to that limitation, Leng et al. $(2016,2019)$ propose the coupling of SEM and the pseudospectral method (Gazdag 1981; Kosloff et al. 1984), which extends the AxiSEM principle to handle more complex 3-D structure (AxiSEM3D). The method can result in a speedup of more than one order of magnitude, depending on model complexity and modeled frequencies.

Conceptually related to AxiSEM, van Driel et al. (2019) propose to use anisotropic adaptive mesh refinements $(a A M R)$ to construct wavefield-adapted meshes for SEM simulations. Making use of prior knowledge on the approximate shape of wavefronts in media with smooth deviations from a potentially discontinuous background, the total number of elements needed to represent the wavefield may be reduced significantly, thereby leading to a decrease in computational cost.

\subsection{Motivation and outline}

The principal motivation of this contribution is a first proof of concept, showing that wavefield-adapted meshes combined with the discrete adjoint technique may lead to an FWI implementation that requires significantly lower computational resources, in cases where variations in medium properties are sufficiently smooth. Indeed, we demonstrate, that under favorable conditions, the approach can result in up to an order of magnitude speedup in both waveform and adjoint modelling, while effectively reducing the frequency $(f)$ scaling from $f^{N+1}$ to $f^{N}$ where $\mathrm{N}$ is the dimension. Though this pilot study is focused on 2-D, it serves the important purpose of algorithmic developments, especially in preparation of extensions to 3-D. Furthermore, it may, as it stands, be applied in scenarios where 2-D wave propagation can serve as an analogue of surface waves in a 3-D Earth.

The manuscript is organized as follows. In section 2, we begin by briefly explaining the underlying idea behind the new meshing technique. section 3 is dedicated to a detailed description of how this fits into the FWI workflow, which additional steps are required, and how we implement them. In sections 4 and 5, we show some examples in both 2-D and 3-D, and demonstrate how this can potentially be used for large scale seismic tomography. Finally, we discuss the most important advantages and limitations of the method, as well as potential future applications.

\section{WAVEFIELD ADAPTED MESHES}

In order to solve a differential equation (e.g., the wave equation) numerically, the continuous fields have to be approximated in a discrete representation. To properly represent a wavefield in a discrete domain, it has to be sampled with a certain number of gridpoints per wavelength; typically around 5-10 gridpoints depending on the method (Fichtner 2010; Igel 2016). The number of gridpoints in a spectral-element mesh is controlled by the number of elements in the mesh and the polynomial order of the basis functions inside the elements. From here on we will assume the most widely used 4th-order polynomial basis functions and focus on the number of elements.

The computational cost of spectral-element simulations directly scale with the number of elements in the discrete mesh. It is thus of great interest to use the minimum number of elements required in each simulation.

In the 2-D example in Fig. 1a), using a polar coordinate system with the pole at the source location, one can compare the wavelengths of the wavefield in the radial and the azimuthal dimensions. Note that by a wavelength in a certain dimension we refer to the distance over which the wavefield repeats itself along the respective dimension. In smooth media, the azimuthal wavelength (perpendicular to the propagation direction) is mostly controlled by medium complexity, and the radial one (parallel to the propagation direction) mostly by the period of the injected source-time-function. Except close to the source, the azimuthal wavelength is much larger than the radial one. This suggests to elongate the elements in azimuthal direction while still keeping an appropriate number of gridpoints per wavelength, thereby greatly reducing the total number of elements in the mesh. Adapting the mesh according to the expected wavefield complexity is called anisotropic adaptive mesh refinement. The following is a summary showing how $a A M R$ meshes work in forward and adjoint wavefield modelling, for more details the reader is referred to van Driel et al. (2019).

Figs 1a) and b) show a wavefield propagating through a homogeneous medium on an $a A M R$ mesh and on a rectilinear mesh, respectively. The elements in the $a A M R$ mesh are aligned with the wavefield propagating from the source location, and the wavefields are approximately identical on both meshes. In the simplistic case of a homogeneous medium, the wavefront and the elements of the $a A M R$ mesh are perfectly aligned. With increasing medium complexity, the wavefield becomes more complex, and so the number of elements in azimuthal direction will need to be increased accordingly, as detailed in section 4.

The $a A M R$ meshes are designed for a single source location. This apparently poses a problem in adjoint simulations where adjoint sources are located at the actual receiver positions. As can be seen in Fig. 1c), the $a A M R$ adjoint wavefield does not resemble a physical wavefield. The adjoint wavefield computed using the rectilinear mesh (Fig. 1d), in contrast, resembles a physical wavefield propagating from the 25 adjoint source locations.

Fortunately, the problem is only an apparent one. In fact, to calculate the gradient, the adjoint wavefield does not have to be a physical wavefield propagating from a point source as illustrated in the discrete adjoint formulation outlined in van Driel et al. (2019). Indeed, Figs 1e) and f) show the shear modulus $(\mu)$ gradients calculated by convolving the time-reversed wavefield of Figs 1a) and b) with the $L_{2}$ waveform misfit, eq. (3) adjoint wavefields of Figs 1c) and d), respectively. Aside from small regions around the receiver locations, the gradients are nearly identical.

Updating the model in FWI is done using the summed gradient 

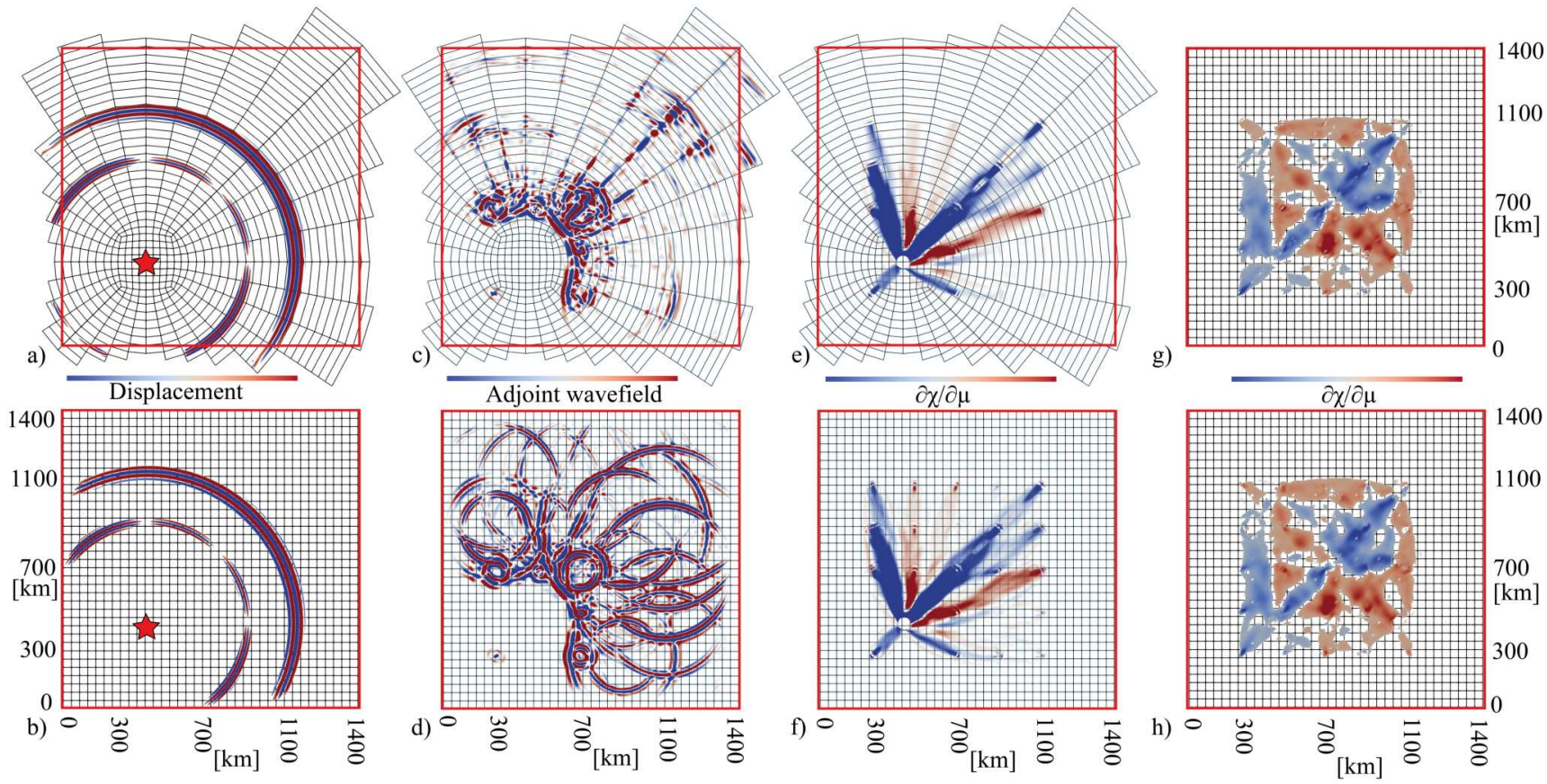

Figure 1. a,b) A snapshot of a forward wavefield propagating outwards from the source location (red star) through a homogeneous medium. The simulations were done using an $a A M R$ mesh and a rectilinear mesh respectively. c,d) Adjoint wavefield propagating from 25 adjoint sources on an $a A M R$ mesh and a rectilinear mesh, respectively. The adjoint source is the derivative of the $L_{2}$ misfit $\chi$, eq. (3), with respect to the synthetics (e.g., Fichtner 2010). e,f) Gradients with respect to shear modulus, $\mu$, calculated by correlating the time-reversed wavefield on panels a) and b) with the adjoint wavefield on panel c) and d), respectively. The displayed gradients are discretized on the Gauss-Lobatto-Legendre (GLL) basis of the meshes. g,h) Sum of gradients for 9 sources. For the $a A M R$ mesh the gradients need to be interpolated to the same mesh prior to summation. The red boxes illustrate equal areas on the different panels. All the colourbars are zero-centered.
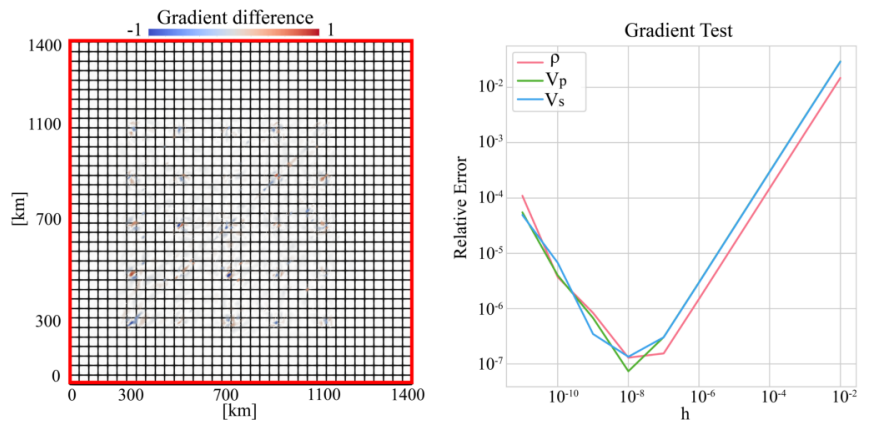

Figure 2. (Left): Difference between the shear modulus gradients in Figs $1 \mathrm{~g}$ ) and $\mathrm{h}$ ). Note that the differences between the gradients are primarily located close to the 25 receivers. The colourbar is normalized based on the gradients on Figs 1g) and h). (Right): Gradient accuracy test using an $a A M R$ mesh for a single source-receiver pair, using eq. (2). Colors represent gradients with respect to $\mathrm{P}$ velocity, $v_{p}, \mathrm{~S}$ velocity $v_{s}$, and density, $\rho$.

from all the simulations used in the respective iteration with the goal of reducing the waveform misfit. The summed gradient from 9 forward and adjoint simulations on the two mesh types are compared in Figs 1g) and h). Each of the 9 aAMR gradients were computed using a custom mesh and were then mapped onto a rectilinear mesh and summed. The gradients are approximately the same and thus result in approximately the same update of the model, at a greatly reduced computational cost in the $a A M R$ case (factor 8.3 speedup).

The difference between the two gradients in Figs 1g) and h) is measured in the left panel of Fig. 2 where one gradient is subtracted from the other. The main discrepancies are at the receiver locations. The discrete gradient test in the right panel of Fig. 2 measures the relative error between the directional derivative and its finite difference approximation, that is,

$\varepsilon=\left|\left(\frac{\chi(\boldsymbol{m}+h \cdot \boldsymbol{m})}{h}-\chi(\boldsymbol{m})\right)-\frac{\partial \chi}{\partial \boldsymbol{m}} \cdot \boldsymbol{m}\right|$,

with the relative error expressed as,

$\operatorname{Rel}(\varepsilon)=\frac{\varepsilon}{\left|\frac{\partial \chi}{\partial \boldsymbol{m}} \cdot \boldsymbol{m}\right|}$,

where $\chi(\boldsymbol{m})$ is the $L_{2}$ waveform misfit, eq. (3) using model $\boldsymbol{m}$, $h$ is the relative model perturbation or finite-difference step length, and $\boldsymbol{m}=\left(v_{p}, v_{s}, \rho\right)$ are the physical model parameters $\mathrm{P}$ velocity, $\mathrm{S}$ velocity, and density, respectively. The relative error plotted as a function of $h$ has a classic hockey stick shape, where larger values of $h$ correspond to linearization errors, and small values of $h$ are related to errors due to numerical precision. This is the expected behaviour of an accurate discrete gradient and demonstrates the correctness of our implementation.

Gradients are discrete representations of continuous sensitivity kernels and the way a gradient looks depends on which basis it is represented on. The gradient test shows that the $a A M R$ gradients are accurate in the $a A M R$ discretization and that the differences to the rectilinear gradients are simply the result of different model parameterization. In this work we demonstrate where this approach is applicable to FWIs and where it starts to break down. 


\section{FULL-WAVEFORM INVERSION WORKFLOW}

The summary in the previous section and the more detailed analysis in van Driel et al. (2019) demonstrate that $a A M R$ is applicable to both forward and adjoint wave equation modelling. In the following section we will detail how to construct a complete full-waveform inversion workflow using these developments.

Complications arise when $a A M R$ meshes are used in FWI. Without the $a A M R$ meshes the model parametrization can be kept on a single inversion mesh, which can be used in both forward and adjoint modelling for all of the (adjoint) sources. Hence, there is no distinction between the discrete representation of the model space for the inversion and the simulations. This makes it trivial to sum different gradients and to update the model.

This procedure is not an option for the $a A M R$ approach since it requires a unique simulation mesh for each source. It is thus a necessity to discretize the model independently of the wavefields. There are multiple options available to store the model parametrization. Using an inversion mesh is a subjective choice of parametrization often used for convenience. The approach used in this study is to apply a rectilinear spectral-element mesh to store the velocity model. In each iteration, the new model is interpolated onto the simulation meshes for the forward and adjoint simulations. Subsequently, the resulting gradients are interpolated back to the inversion mesh. The complete workflow is illustrated in a flowchart in Fig. 3. The additional steps in the workflow which are required to use the $a A M R$ meshes are explained in more detail in the coming sections.

\subsection{Building meshes for individual sources}

One of the main extensions to standard FWI is that each source requires a unique mesh. The meshing only needs to be carried out once per source, and so it does not impose a significant computational burden.

In 2-D, each mesh is created by meshing the domain with a regular grid in polar coordinates. The origin of the coordinate system is placed at the source location. To avoid singular elements, a regular grid is built around the source location, replacing the polar grid in a small region. The radial dimension of the polar grid is discretized based on the minimum modelled period. The discretization in the azimuthal dimension is, however, a free parameter governed by i) the complexity of the medium and ii) how well a circular wavefield needs to be approximated. The latter can be achieved with fewer elements if the shapes of the elements can follow higher-order polynomials, rather than being restricted to straight lines. The number of elements in the azimuthal direction is be kept constant, as a function of radius, as seen in Fig. 1. This is, however, not a restriction, as will be demonstrated in section 5 .

\subsection{Interpolation}

When an inversion discretization, i.e. an inversion mesh or a Fourier basis, is used for storage and representation of the medium, interpolation to and from the various $a A M R$ meshes becomes necessary. The main objective when defining the operator which interpolates from the inversion discretization onto the simulation meshes is to preserve the properties of the medium which the wavefield is sensitive to, that is, the effective medium. Once the interpolation operator is defined, the adjoint interpolation operator is used to map the computed gradients back to the inversion discretization. In the numerical examples studied in section 4 , a pointwise evaluation of the GLL-basis of the model on the inversion mesh is sufficient to produce an $a A M R$ representation for numerical simulations. However, this may not generally be sufficient, as we will further discuss in section 6.3.

\section{NUMERICAL EXPERIMENTS}

To test the method, we constructed a 2-D synthetic example with a homogeneous, isotropic starting model and attempted to reconstruct a random medium with up to $\pm 10 \% \mathrm{P}$ and $\mathrm{S}$ velocity $\left(v_{p}\right.$ and $v_{s}$ ) deviations from the initial model. The random target medium was computed using the Fourier method (Igel \& Gudmundsson 1997; Meschede \& Romanowicz 2015).

\subsection{Experimental Setup}

The domain is $1400 \mathrm{~km}$ by $1400 \mathrm{~km}$ wide, with 9 sources distributed regularly between $400 \mathrm{~km}$ and $1000 \mathrm{~km}$ in each direction. Receivers are placed every $100 \mathrm{~km}$ in an $800 \mathrm{~km}$ by $800 \mathrm{~km}$ region. The source-receiver distribution is illustrated in Fig. 4. The sources are all moment tensor sources, and artificial data is calculated using a regular grid rectilinear mesh designed to resolve $5 \mathrm{~s}$ period elastic waves. The source time function is a Ricker wavelet, and each source was simulated for $250 \mathrm{~s}$ using the spectral-element wave propagation solver Salvus (Afanasiev et al. 2019). We conducted the synthetic inversion experiment using the proposed workflow with $a A M R$ meshes which have a varying number of elements in azimuthal direction.

\subsection{Forward Modelling Validation}

We analyzed forward modelling errors through the true medium and compared the results from the various meshes used in the experiment. The wavefield was sampled at 80 regularly spaced receivers. At each receiver, the absolute maximum amplitude calculated on the rectilinear mesh was used as a normalization factor prior to misfit calculation. The normalization was done to make each receiver and each source equally important, removing the effect of source-receiver distance and magnitude on the misfit. The $L_{2}$ waveform error was computed component wise,

$\chi\left(\boldsymbol{d}_{\text {obs }}, \boldsymbol{d}_{\text {pred }}\right)=\frac{1}{2} \int_{T}\left[\frac{\boldsymbol{d}_{\text {obs }}(t)-\boldsymbol{d}_{\text {pred }}(t)}{\max \left|\boldsymbol{d}_{\text {obs }}\right|}\right]^{2} d t$,

where $\boldsymbol{d}_{o b s}$ is the artificial data, $\boldsymbol{d}_{\text {pred }}$ is the synthetic data and $T$ is the time window, the error was averaged across the receivers. To test the effect of medium complexity, we simplified the true medium through filtering. For this, we performed a 2-D Fourier transform to obtain the wavenumber domain medium, and then multiplied the spectrum with a centered flat circular filter with a Gaussian taper at the edges. An inverse 2-D Fourier transform was used to acquire the filtered medium. The maximum wavenumber (complexity) existing in the new media was controlled by varying the extent of the circular filter in the wavenumber domain.

We ran $250 \mathrm{~s}$ long simulations through the media of varying complexity and compared the computed errors for the varying $a A M R$ meshes. The results are displayed in Fig. 5. It shows how the meshes with 48 or more azimuthal elements perform similarly well for the unfiltered medium but as the medium becomes less complex, the mesh with 32 elements achieves similar accuracy 


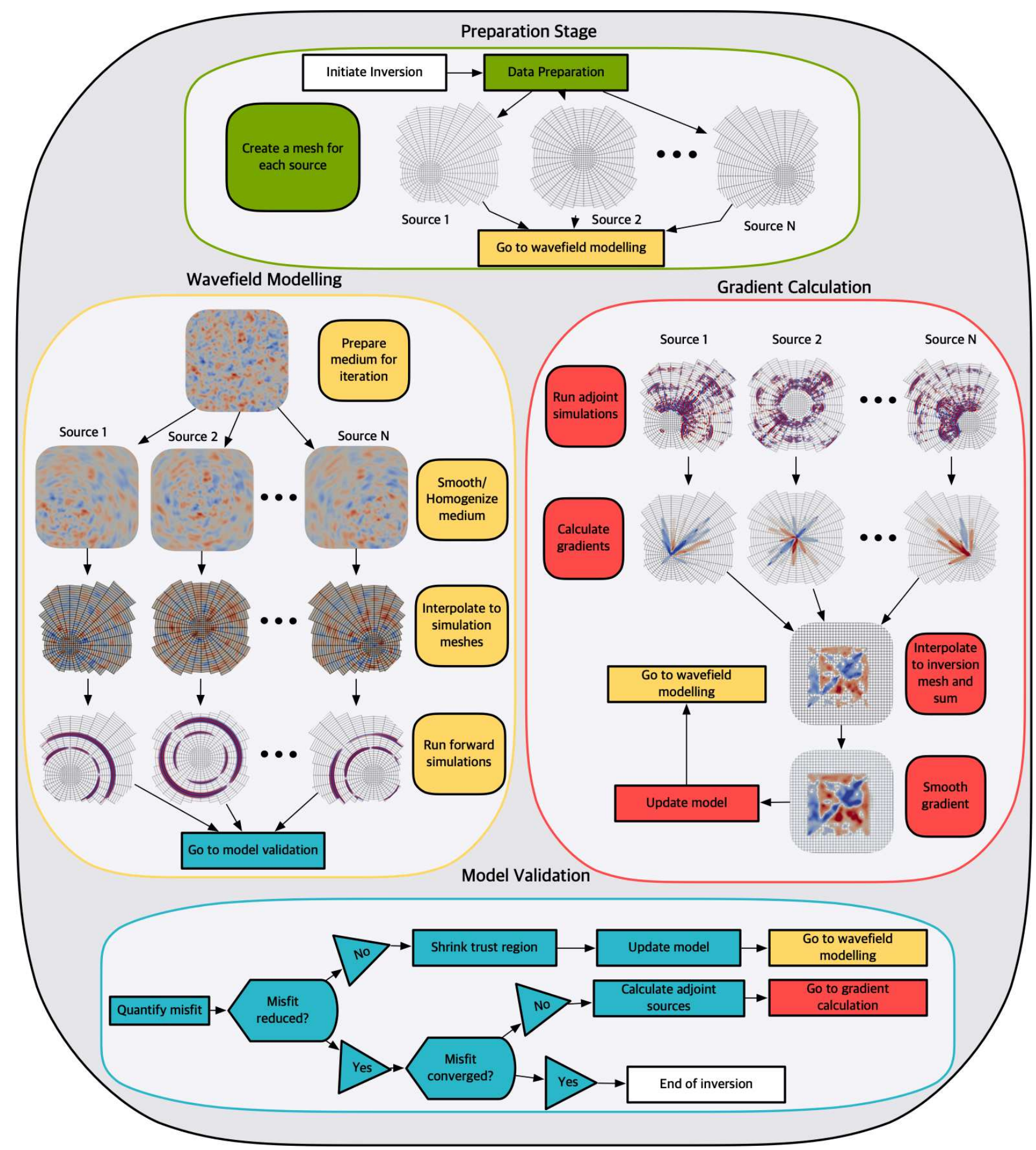

Figure 3. A flowchart explaining the trust-region L-BFGS (Nocedal \& Wright 2006) FWI workflow using $a A M R$ meshes. The medium smoothing is exaggerated for visualization. More details regarding smoothing/homogenization can be found in the discussion section 6.3. A similar workflow can be implemented for any other gradient descent algorithm.

as the others. The mesh with 24 azimuthal elements gets close to the others as the medium becomes simpler, but the 16 azimuthal element mesh does not get below an order of magnitude higher errors than the others in spite of the increasing simplification of the medium. That indicates that the dominating factor in the errors associated with the 16 azimuthal element mesh is the limitation of the linear element shapes and how well they can approximate a circular wavefield. 


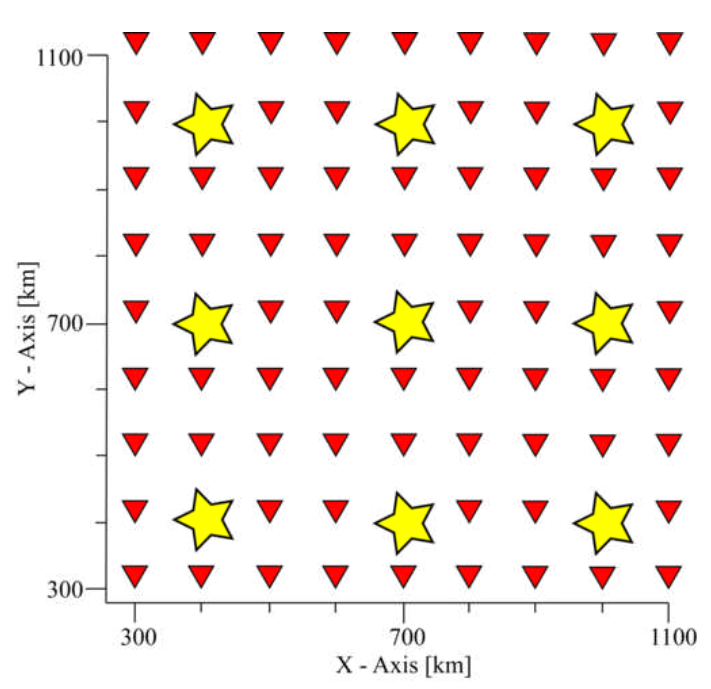

Figure 4. An illustration of the distribution of sources (stars) and receivers (inverse triangles). Each source is recorded by 80 receivers (all except the one on top of the respective source). A $300 \mathrm{~km}$ wide buffer on each side is used for absorbing boundaries.

\subsection{Inversion}

As a baseline, we inverted for the random medium starting from a homogeneous model using the conventional approach with the same rectilinear grid as was used to calculate the artificial data (noise free). The mesh contains 20,736 elements and we did 30 trust-region L-BFGS (Liu \& Nocedal 1989; Nocedal \& Wright 2006) iterations, reducing the waveform misfit in each iteration. We simultaneously inverted for shear modulus, $\mu$, and the Lamé parameter $\lambda$, keeping density, $\rho$, constant. We compare the shear modulus models using the $L_{2}$ model misfit

$\chi\left(M_{1}, M_{2}\right)=\int_{\Omega}\left[M_{1}(\boldsymbol{x})-M_{2}(\boldsymbol{x})\right]^{2} d \boldsymbol{x}$,

where $M_{1}$ and $M_{2}$ are two separate models, $\boldsymbol{x}$ is a point in the models, and $\Omega$ is the area of the models. After the last iteration, the $L_{2}$ model misfit had been reduced by $88 \%$ compared to the initial model and the $L_{2}$ waveform misfits, eq. (3) compared to the artificial data had been reduced by $99.6 \%$ from the starting model.

To test the new method we attempted the same type of recovery for various versions of the $a A M R$ meshes. We varied the num-

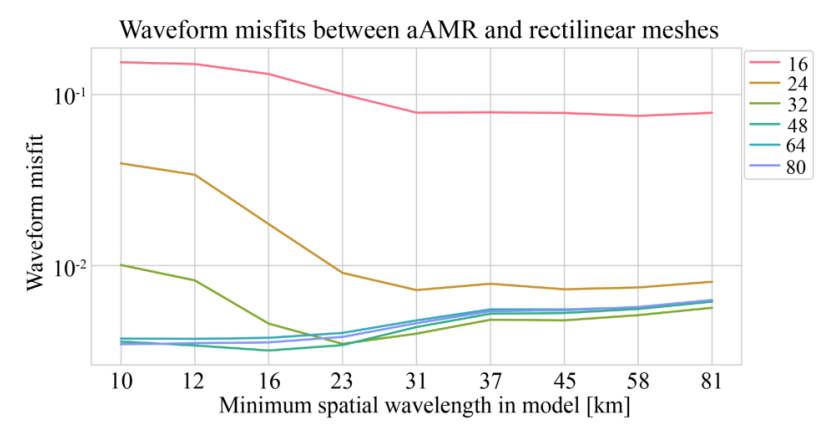

Figure 5. Average $L_{2}$ forward modelling errors, eq. (3) between various $a A M R$ meshes and the rectilinear mesh using the random target medium. The medium is filtered in the wavenumber domain to remove the highwavenumber structure. Note the logarithmic scale on the vertical axis. The legend refers to the number of elements in azimuthal direction.
Table 1. A comparison of Model Misfit (MM) between final model and true model, normalized by misfit between homogeneous starting model and final model, Waveform Misfit Reduction (WMR), the Simulation Time (ST) used to achive these misfit reductions and the relative speedup achieved by using the $a A M R$ meshes (SU) with the same timestep. The Rect mesh is the classic method while the numbers in the Mesh column represent the azimuthal elements in the $a A M R$ meshes.

\begin{tabular}{rrrrr}
\hline Mesh & MM [\%] & WMR [\%] & ST [CPUhrs] & SU \\
\hline Rect & 12 & 99.66 & 66.67 & - \\
8 & 1318 & 27.64 & 2.62 & 25.45 \\
16 & 125 & 88.24 & 4.35 & 15.33 \\
24 & 26 & 97.87 & 6.20 & 10.75 \\
32 & 16 & 99.03 & 8.07 & 8.26 \\
48 & 14 & 99.45 & 11.63 & 5.73 \\
64 & 12 & 99.57 & 15.23 & 4.38 \\
80 & 12 & 99.58 & 18.57 & 3.59 \\
\hline
\end{tabular}

ber of elements used to cover the azimuthal circumference around the source from 8 to 80 . The number of elements in the respective meshes varied from 820 to 5782 . We analysed the quality of model recoveries as a function of simulation cost and iterations (Fig. 6). The actual value of the computational speedup is problemdependent and correlates with the size of the domain and the maximum frequency, while it is anti-correlated with medium complexity.

As Fig. 6 shows, increasing the number of azimuthal elements above 32 does not lead to significant improvements in model reconstruction and mostly leads to an increase in computational load (see Table 1). All of the meshes with 32 azimuthal elements or above manage to reduce the model misfit by roughly the same amount as the regular FWI. The waveform misfit reductions vary from $99.0 \%$ to $99.6 \%$, and the model misfit reductions vary from $84 \%$ to $88 \%$. The computational gain achieved by using the $a A M R$ meshes is visualized on the left panel of Fig. 6, where the evolution of the model misfit is plotted as a function of simulation time.

The reconstructed models along with the respective meshes can be seen in Fig. 7. The differences between the rectilinear reconstruction and the $a A M R$ reconstructions, which use 48 elements or more, are visually indistinguishable. For 24-32 azimuthal elements, the reconstruction looks similar to the rectilinear one. For fewer azimuthal elements the final images start to deviate significantly from the rectilinear recovery. The quantitative analysis of the model differences in Fig. 8 supports that observation and reveals that the 16 azimuthal element reconstruction mostly catches the large-scale features of the model but not the fine-scale details. The other models, however, capture both the fine- and the large-scale features.

Fig. 5 reveals that the errors in model reconstruction, seen in Figs 7 and 8, correspond to the forward modelling errors for the different $a A M R$ meshes through the true medium. This indicates that the errors in the reconstructions relate to the errors in the forward wavefield and interpolations between grids (steps 3 and 4 in the wavefield modelling box and step 3 in the gradient calculation cox in Fig. 3).

\section{TOWARDS 3-D}

While this proof of concept is focused on 2-D, the longer-term goal is a 3-D global-scale application, already hinted at by the 3-D forward modelling experiments of van Driel et al. (2019). Here, we 

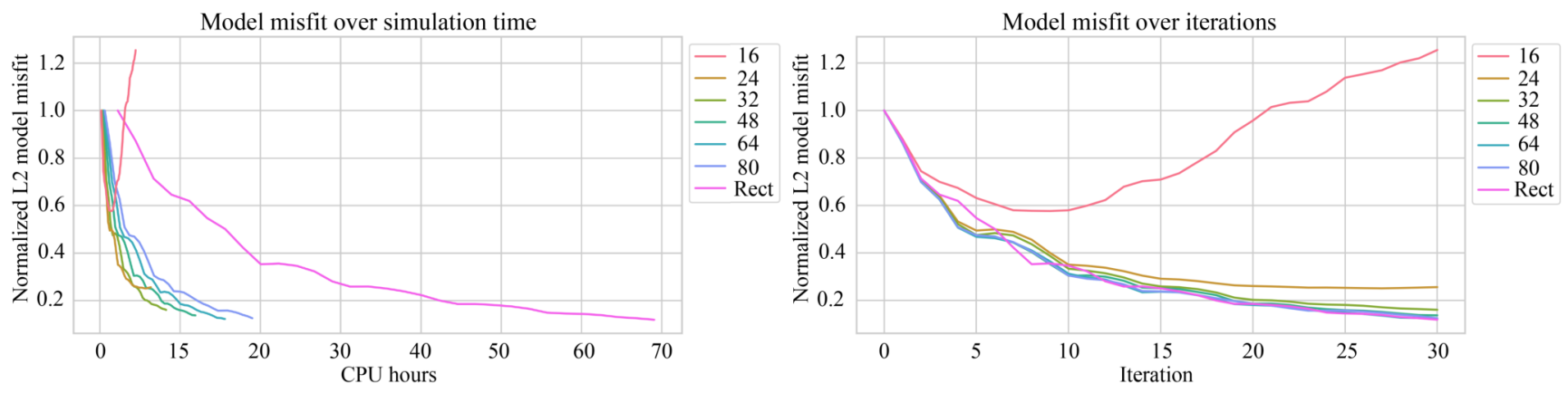

Figure 6. (Left): $L_{2}$ model misfit, eq. (4), as a function of simulation time in the FWI. The model misfit is normalized by the $L_{2}$ model misfit of the homogeneous starting model. (Right): The same misfit measurement but as a function of L-BFGS iterations.

provide a first step in this direction in the form of global-scale kernel calculations.

The extension of the $a A M R$ meshing to 3-D is conceptually related to the AxiSEM approach (Nissen-Meyer et al. 2008). Given that we are designing a mesh to represent a wavefield propagating away from a source at the North Pole, a D-shaped AxiSEM mesh is extruded, rotating around a vertical axis through the poles. To avoid singular elements at the symmetry axis, a number of elements around the axis are removed and replaced by a cylinder which is meshed as disc which is extruded between the poles. The discretization in the radial and polar directions is controlled by the frequency which needs to be resolved, while the discretization in the azimuthal direction is, similar to the 2-D case, controlled by the medium/source complexity and the need to approximate a sphere accurately. If the source is located at some other location than one of the poles, the discretization is rotated in order to make the cylinder go through the source location. Fig. 9c) shows a 3-D global $a A M R$ mesh with 4th-order shape maps of the elements (van Driel et al. 2019, section 4.2). The surface elements are refined $30^{\circ}$ from the source location to resolve surface waves better. This has the additional benefit of better point approximations for receiver locations.

We computed a single source-receiver pair gradient on a standard global cubed sphere mesh, as well as on a 3-D $a A M R$ mesh. These are shown in Figs 9a) and d), respectively. The meshes are designed to resolve a minimum period of $50 \mathrm{~s}$ at 2 elements per minimum wavelength, and they both have a 1-D isotropic velocity model (Dziewonski \& Anderson 1981). The cubed sphere has 1,699,840 elements, while the $a A M R$ mesh has 120,416. Representative slices through the $\mathrm{P}$ velocity gradients of the first-arrival $\mathrm{P}$-wave traveltime are displayed in Figs 9b) and c). Due to the differences in model parameterization, the gradients, that is, the projection of sensitivity kernels onto the different basis functions, do not look identical. However, as discussed in section 2, both are correct discrete gradients corresponding to their respective parametrizations. Similar to the 2-D case, the main difference is near the receiver locations. As demonstrated in the numerical example, combining many of these gradients should conclude the iteration with approximately the same model update at a greatly reduced computational cost. Computing the $a A M R$ gradient was over 14 times cheaper computationally, meaning that one can perform roughly 14 iterations at the cost of one, by using $a A M R$ meshes.

\section{DISCUSSION AND CONCLUSIONS}

We presented a first proof of concept, showing that wavefieldadapted meshes can be used in an FWI workflow, potentially leading to significant computational savings. In the following paragraphs, we discuss limitations of the method, current and future domains of applicability, as well as further technical issues such as homogenization for improved interpolation, and the effective frequency scaling.

\subsection{Limitations of this approach}

The usage of $a A M R$ meshes is an add-on to spectral-element methods. The applicability and potential speedups depend on the specific problem and whether it meets the assumptions of the $a A M R$ meshes. It is thus essential to clearly outline the niche where this approach is likely to be beneficial. For this, we recall that the concept of wavefield-adapted meshes rests on the assumption that our prior knowledge on the geometry of wavefronts and complexity of the wavefield can be approximated by the mesh.

In 2-D, this is the case, for instance, when the medium is sufficiently smooth to avoid significant scattering. A comparison of the usage of 2-D $a A M R$ meshes in a smooth medium versus a medium with a sharp discontinuity is shown in Figs 11 and 10. It demonstrates that the approach requires smooth media because scattering breaks the approximate azimuthal symmetry of the wavefield.

In 3-D and at regional to global scales, the Earth is roughly spherically symmetric. The reflected waves from the global internal discontinuities preserve the azimuthal symmetry; a fact that is exploited by the AxiSEM approach (Nissen-Meyer et al. 2007, 2008, 2014; Leng et al. 2016, 2019). Thus, approximately spherical discontinuties do not pose a problem for $a A M R$ meshes in 3-D. Similar to 2-D, the deviations from the approximately spherically symmetric background must, however, be smooth enough to avoid significant off-great-circle scattering. The precise meaning of 'significant' depends on the actual data one wishes to exploit, and therefore must be assessed on a case-by-case basis, e.g., using a careful forward modelling study along the lines of Fig. 5.

\subsection{Current and future domains of applicability}

Despite being a basic feasibility study in 2-D, this work has practical applicability. From a methodological perspective it serves the purpose of algorithmic developments, concerning, for instance, interpolation between different meshes, workflow management, and the nonlinear optimization scheme. From an application perspective, the 2-D approach may be used in cross-hole seismology, or 


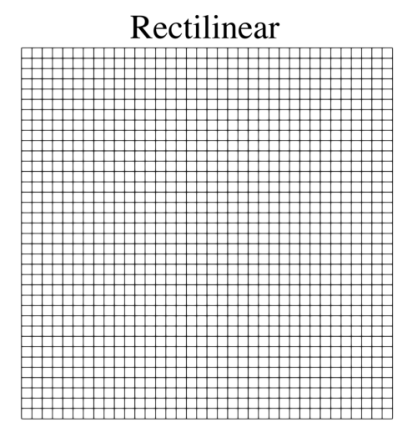

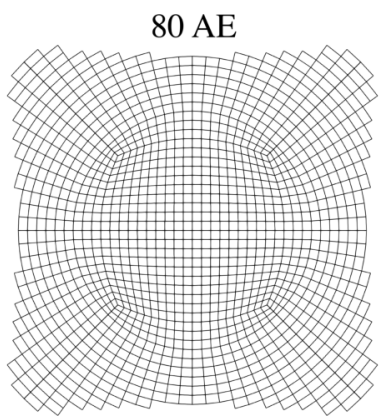

$64 \mathrm{AE}$
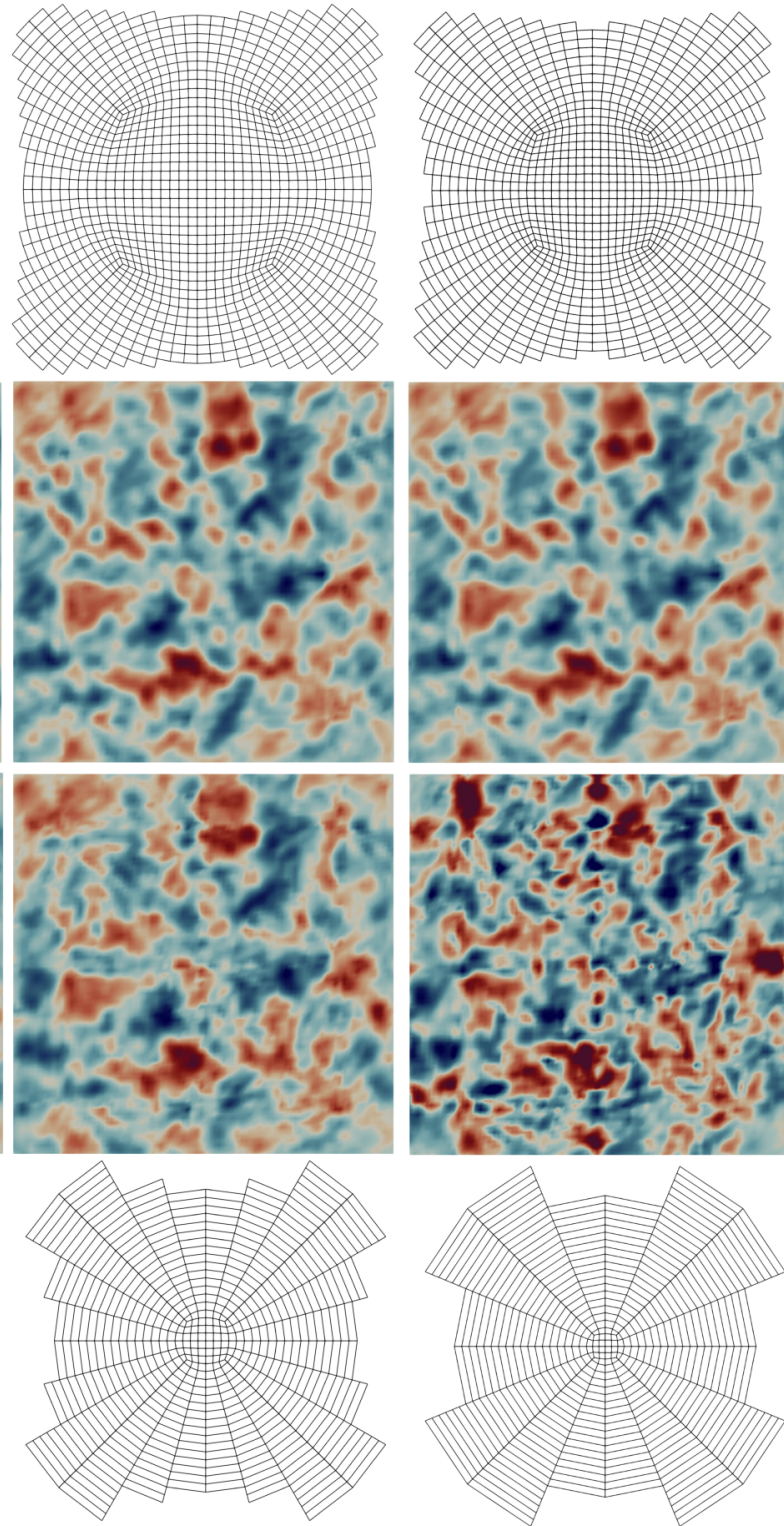

$24 \mathrm{AE}$
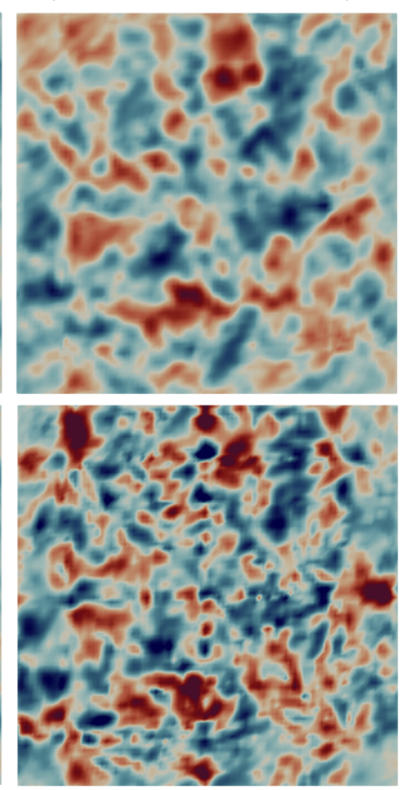

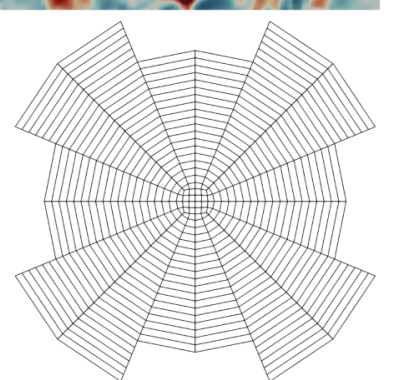

$16 \mathrm{AE}$
$48 \mathrm{AE}$
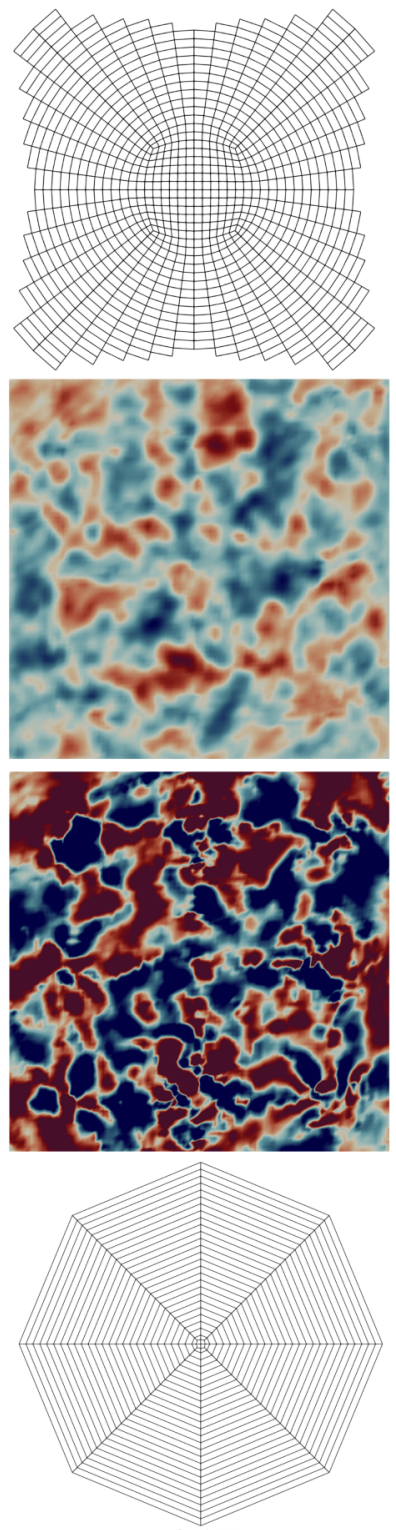

$8 \mathrm{AE}$

\section{$3.7 \cdot 10^{10}$}

$\mu[\mathrm{Pa}]$

$4.2 \cdot 10^{10}$

Figure 7. Comparison of FWI inversion results and corresponding simulation meshes, varying the number of azimuthal elements (AE). In the $a A M R$ case, only the mesh with the source at the center is displayed for simplicity. For easier visualization, the displayed meshes have a coarser discretization (factor 4) in the radial direction than the ones that were actually used in the inversion, but the same discretization in the azimuthal direction. The rectilinear mesh has a coarser discretization (factor 4) in both dimensions in the mesh visualization. Furthermore, in the displayed aAMR discretizations, the rectilinear source region is larger than on the meshes that were used in the inversion. The plotted regions are the same as for the receiver coverage displayed Fig. 4.

in cases where 2-D wave propagation can serve as an analogue for surface wave propagation in the 3-D Earth (e.g., Peter et al. 2007, 2009).

The FWI workflow presented in section 3 is essentially a generalization of conventional workflows. As the medium complexity is always known beforehand, the meshes can be adjusted based on the medium of each iteration and in the most extreme case, the fallback option is to use conventional meshes. The computational cost of the additional steps in the presented workflow compared to a conventional one is negligible, so in a case where conventional meshes need to be used, the presented workflow does not add a considerable computational cost.

Moreover, the workflow is not tied to $a A M R$ meshes, but also applies to inversions, where each source is is modelled on a different subsection of the domain (e.g. when the computational mesh is truncated to the source-receiver geometry of each simulation). 

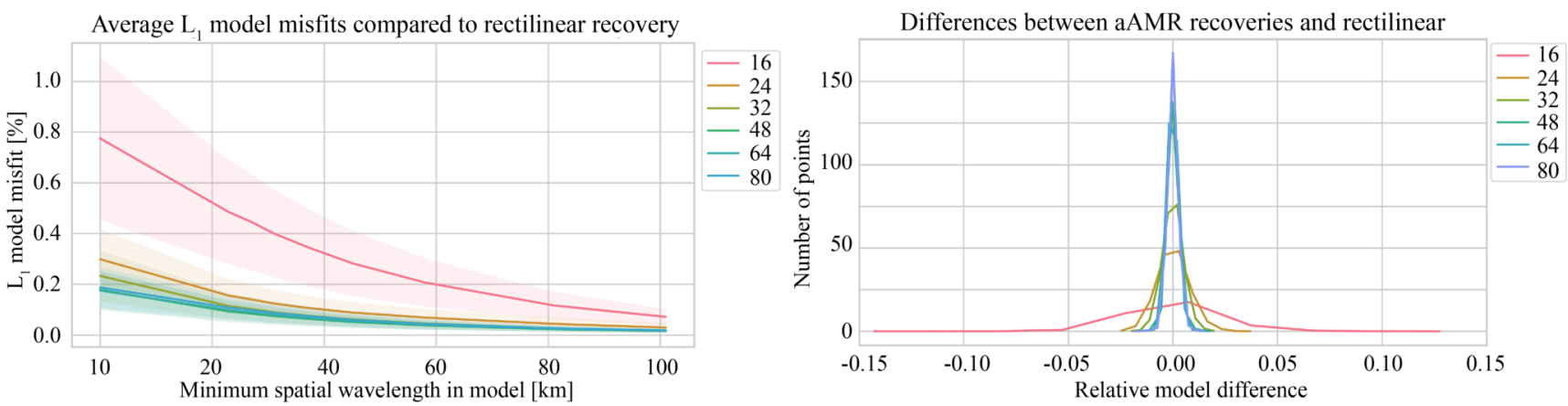

Figure 8. (Left): Mean of pointwise absolute model misfits between rectilinear recovery and various $a A M R$ recoveries. The misfits are normalized by the shear modulus of the homogeneous medium to make the misfit relate to deviations from the homogeneous medium. The true model and the homogeneous model would have an average absolute misfit of about $2 \%$. The models are lowpass filtered in the wavenumber domain, the horizontal axis displays the minimum spatial wavelength in the filtered model. The shaded regions represent half a standard deviation. (Right): The histogram of the pointwise model misfit measurements for the unfiltered rectilinear model.

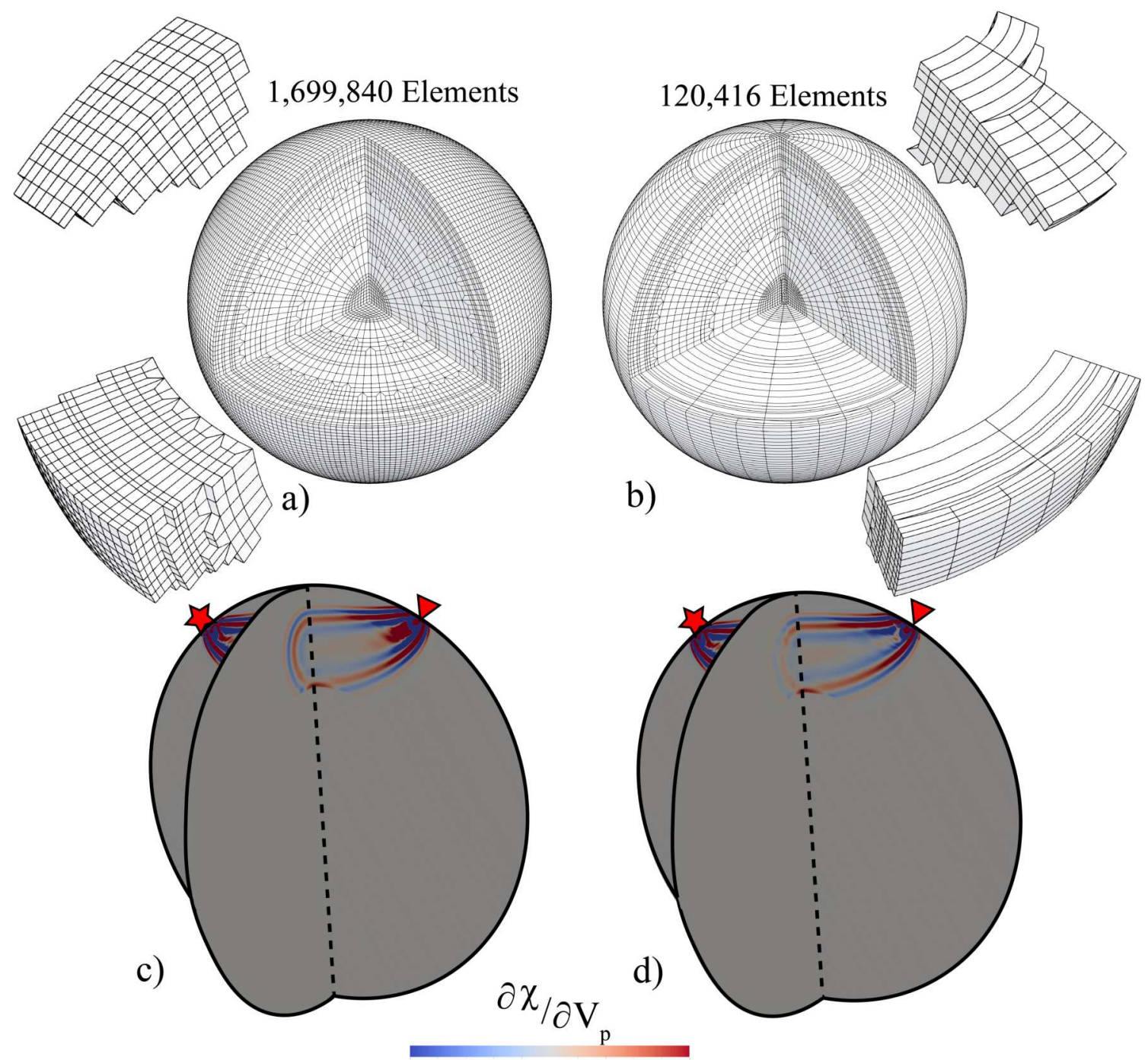

Figure 9. a): A typical global 3-D cubed sphere hexahedral mesh (Ronchi et al. 1996) where element dimensions are controlled by the s-wave velocity. b): A global 3-D $a A M R$ mesh with the radial and the polar dimensions identical to the cubed sphere mesh, but elongated elements in azimuthal direction. Also note the cylindrical mesh along the axis that avoids ill-shaped elements (similarly to the 2-D examples) and the azimuthal refinements for better resolution of surface waves. c,d): Bodywave gradients calculated from a single source-receiver pair on meshes a) and b), respectively. Note that, as in the 2-D case, the main difference is close to the receiver location (triangle). 

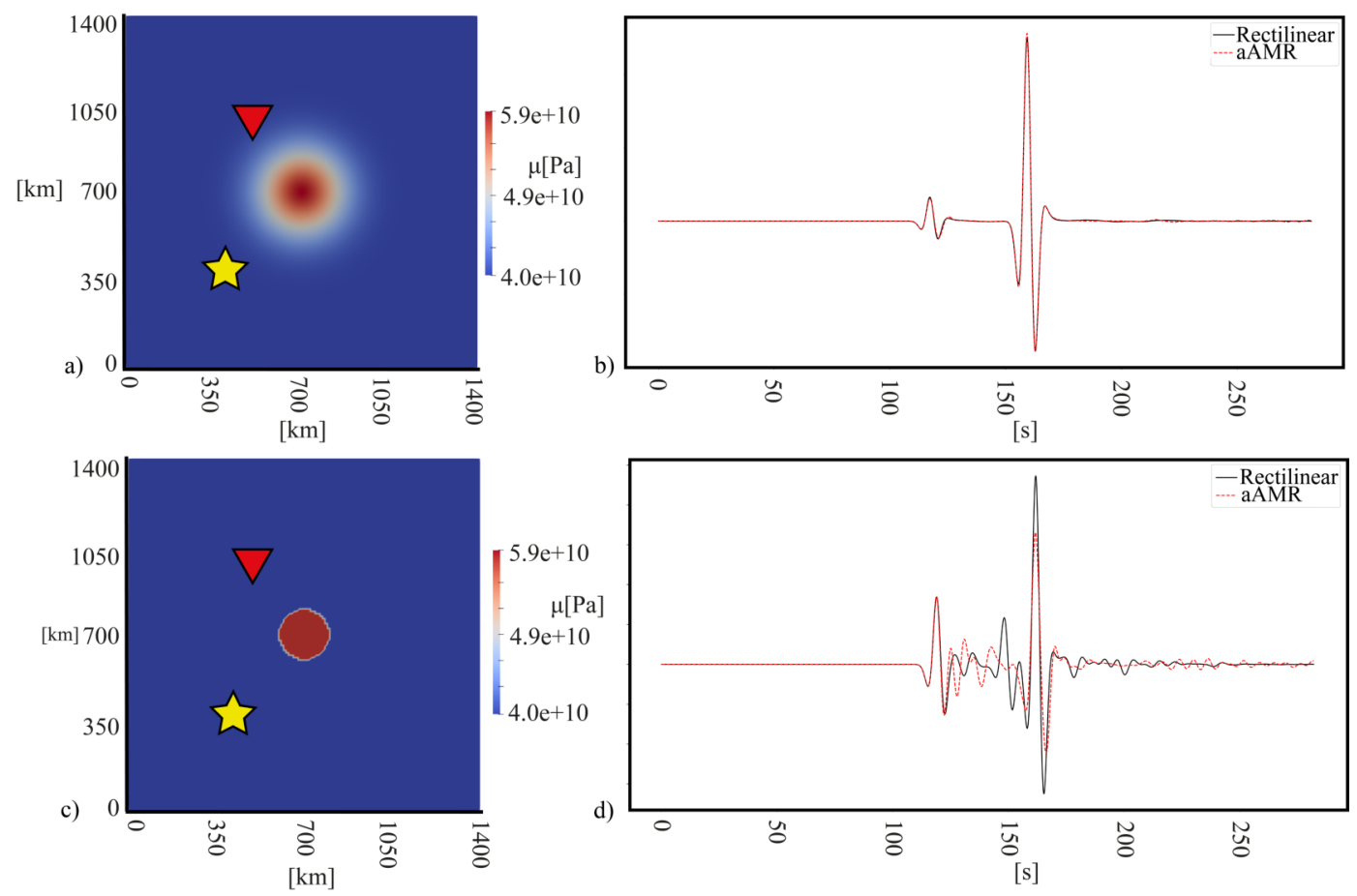

Figure 10. Example for the effect of medium roughness on $a A M R$ modelling accuracy. a): A homogeneous medium with a Gaussian-shaped anomaly in shear modulus with $200 \mathrm{~km}$ diameter and $50 \%$ amplitude. b): The waveform recorded on a station located at the red triangle from a source at the yellow star. The comparison is between a rectilinear mesh and an $a A M R$ mesh with 32 elements in azimuthal direction. c,d): Same for a model with a sharp boundary of the anomaly. The $a A M R$ meshes work much better for the smooth model as the reflections from the sharp boundary break the basic assumption of the wave fronts' alignment with the long side of the elements

\subsection{Homogenization prior to interpolation}

The simulation meshes have, like the wavefields, variable spatial resolution. Smaller-scale structure in some regions may thus be well represented on the fine inversion mesh but less so on a potentially coarser simulation mesh. This under-sampling may result in spatial aliasing when the model is interpolated from the inversion onto the simulation meshes. To ensure that the wavefield 'sees' the effective medium in both discretizations, the model should ideally be homogenized for each simulation mesh prior to interpolation. Several homogenization approaches for wave propagation have been developed in recent years (e.g., Capdeville \& Marigo 2007; Capdeville et al. 2013; Fichtner \& Hanasoge 2017; Cupillard \& Capdeville 2018). An approximate alternative to homogenization is space-dependent anisotropic smoothing, where, in our specific case, the medium is smoothed primarily in azimuthal direction. This is visualized as the second step in the wavefield modelling box in Fig. 3. The reasoning behind space-dependence and anisotropy of the smoothing is that the element edge lengths vary both as a function of space and dimension, and the risk of spatial aliasing is a function of model complexity and element edge lengths. Though we expect homogenization or smoothing to become relevant in future applications, we empirically found that straightforward sampling of the medium on the Gauss-Lobatto-Legendre points was sufficient for the examples presented in section 4 .

\subsection{Frequency scaling}

An important feature of the $a A M R$ meshes is how the number of required elements scales with frequency. As mentioned in the introduction, the number of elements required in the current standard meshes, scales with frequency to the power of their respective dimension $(N)$. The aAMR meshes, however, have one dimension which is quasi-independent of frequency. The number of elements thus scale approximately with frequency to the power of $N-1$. Fig. 12 illustrates this relation. The difference in frequency scaling indicates that the $a A M R$ meshes are a key development in order to reach higher frequencies in large-scale inversions. It is however important to note that with increasing the frequency, the azimuthal complexity of the wavefield increases, and thus the frequency scaling of the $a A M R$ meshes is medium dependent. Unfortunately a theoretical relation between frequency and azimuthal complexity of the wavefield does not exist. It has, however, been investigated numerically by Leng et al. $(2016,2019)$ where it was found that in a period range where global seismic tomographic models currently exist, 34 to $5 \mathrm{~s}$, the frequency has a minimal effect on the azimuthal complexity of the wavefield.

\section{ACKNOWLEDGMENTS}

This work was supported by the European Research Council (ERC) under the EU's Horizon 2020 programme (grant No. 714069). We gratefully acknowledge support from the Swiss National Supercomputing Center (CSCS) in the form of computing time grants c13 and s868. Data visualizations were done using Paraview (Ahrens et al. 2005) and Matplotlib (Hunter 2007), data processing was done using Numpy (Oliphant 2006), Obspy (Beyreuther et al. 2010) and ASDF (Krischer et al. 2016). The inversion workflow was developed using Jupyter Notebooks (Kluyver et al. 2016). 
Waveform error as a function of smoothness of perturbation

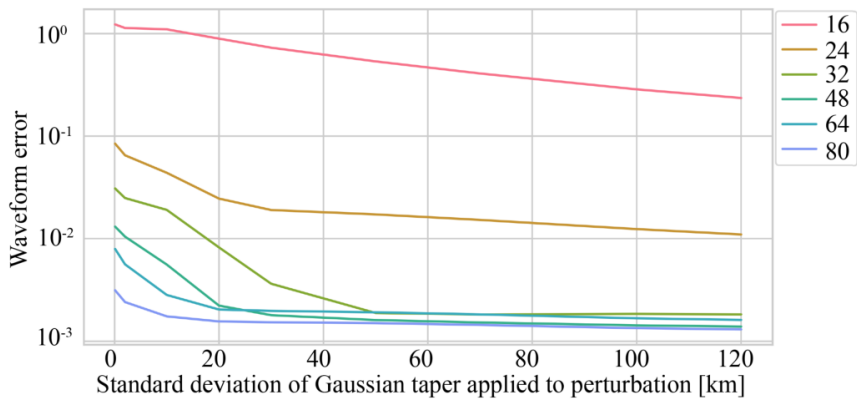

Waveform error as a function of strength of perturbation

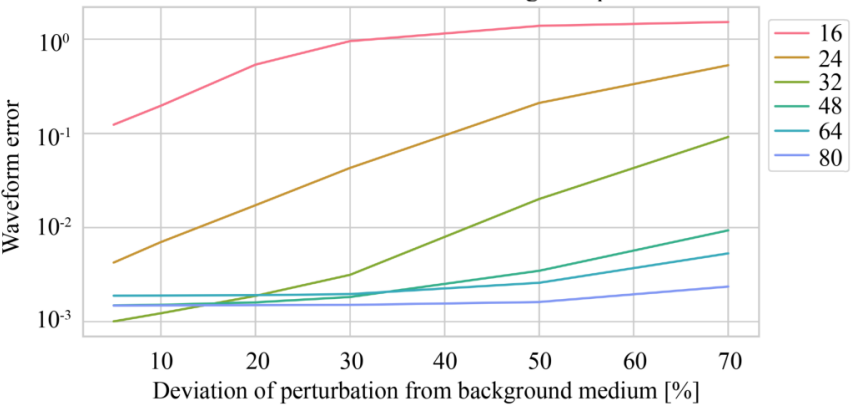

Figure 11. Quantifications of waveform misfits for similar models as displayed in Fig. 10, with stations distributed around the anomaly. (Left): Waveform misfits for a homogeneous model with a $20 \%$ perturbation, as a function of smoothness of the perturbation. Smoothness of the perturbation is defined here as the standard deviation of the Gaussian taper applied to it. (Right): Waveform misfits from a homogeneous model with a perturbation with a $50 \mathrm{~km}$ Gaussian taper applied to it as a function of anomaly strength. Note the log scale on both plots. The ground truth was calculated on a very fine rectilinear mesh.
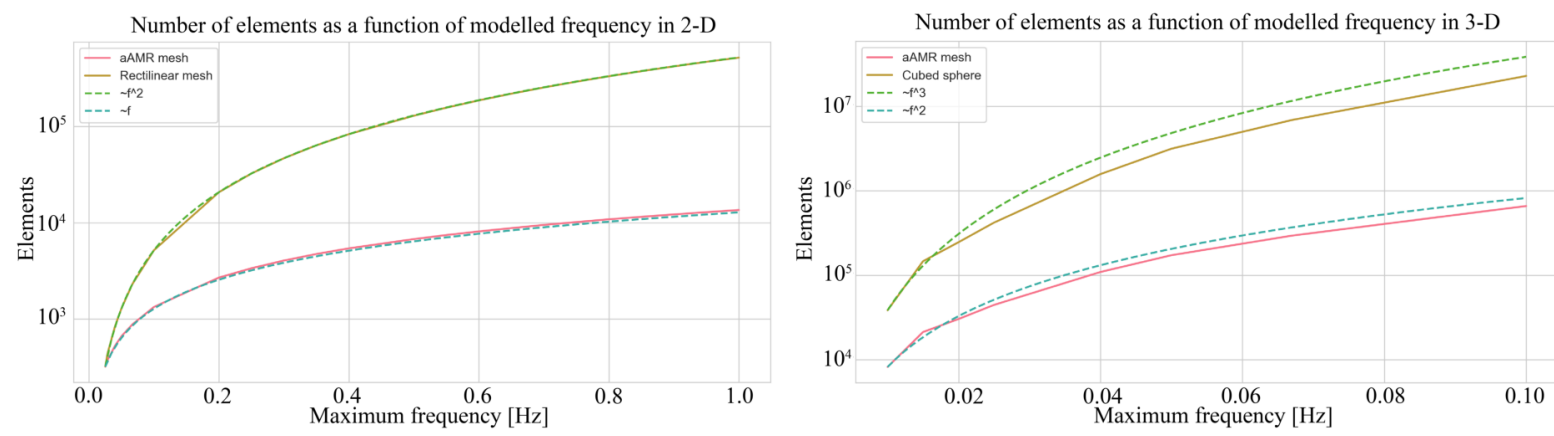

Figure 12. (Left): The number of elements required to mesh a certain medium in 2-D compared to the theoretical frequency scaling laws. (Right): Same measurement in 3-D. Overall it can be seen that $a A M R$ meshes scale with frequency to the power of $N-1$ compared to the scaling of the current standard meshes to the power of $N$. In both 2-D and 3-D, these results assume a constant azimuthal resolution of the wavefield. 


\section{REFERENCES}

Afanasiev, M., Boehm, C., van Driel, M., Krischer, L., Rietmann, M., May, D. A., Knepley, M. G., \& Fichtner, A., 2019. Modular and flexible spectral-element waveform modelling in two and three dimensions, Geophys. J Int., , 216(3), 1675-1692.

Ahrens, J., Geveci, B., \& Law, C., 2005. Paraview: An end-user tool for large data visualization, The visualization handbook, 717.

Aki, K., Christoffersson, A., \& Husebye, E. S., 1977. Determination of the three-dimensional seismic structure of the lithosphere, J. geophys. Res., , 82(2), 277-296.

Bamberger, A., Chavent, G., Hemons, C., \& Lailly, P., 1982. Inversion of normal incidence seismograms., Geophysics, , 47, 757-770.

Beyreuther, M., Barsch, R., Krischer, L., Megies, T., Behr, Y., \& Wassermann, J., 2010. Obspy: A python toolbox for seismology, Seismological Research Letters, 81(3), 530-533.

Boehm, C., Martiartu, N. K., Vinard, N., Balic, I. J., \& Fichtner, A., 2018. Time-domain spectral-element ultrasound waveform tomography using a stochastic quasi-newton method, in Medical Imaging 2018: Ultrasonic Imaging and Tomography, vol. 10580, p. $105800 \mathrm{H}$, International Society for Optics and Photonics.

Bozdağ, E., Peter, D., Lefebvre, M., Komatitsch, D., Tromp, J., Hill, J., Podhorszki, N., \& Pugmire, D., 2016. Global adjoint tomography: firstgeneration model, Geophys. J Int., , 207(3), 1739-1766.

Capdeville, Y. \& Marigo, J.-J., 2007. Second order homogenization of the elastic wave equation for non-periodic layered media, Geophys. J Int., , 170(2), 823-838

Capdeville, Y., Stutzmann, E., Wang, N., \& Montagner, J.-P., 2013. Residual homogenization for seismic forward and inverse problems in layered media, Geophys. J Int., , 194(1), 470-487.

Chen, P., Jordan, T. H., \& Zhao, L., 2007a. Full 3D waveform tomography: a comparison between the scattering-integral and adjoint-wavefield methods, Geophys. J Int., , 170, 175-181.

Chen, P., Zhao, L., \& Jordan, T. H., 2007b. Full 3D tomography for the crustal structure of the Los Angeles region., Bull. seism. Soc. Am., , 97, 1094-1120.

Courant, R., Friedrichs, K., \& Lewy, H., 1928. Über die partiellen differenzengleichungen der mathematischen physik, Mathematische annalen, 100(1), 32-74

Cupillard, P. \& Capdeville, Y., 2018. Non-periodic homogenization of 3D elastic media for the seismic wave equation, Geophys. J Int., , 213 983-1001.

Cupillard, P., Delavaud, E., Burgos, G., Festa, G., Vilotte, J.-P., Capdeville, Y., \& Montagner, J.-P., 2012. Regsem: a versatile code based on the spectral element method to compute seismic wave propagation at the regional scale, Geophys. J Int., , 188(3), 1203-1220.

Dumbser, M., Käser, M., \& Toro, E., 2007. An arbitrary high-order discontinuous Galerkin method for elastic waves on unstructures meshes, Part V: Local time stepping and p-adaptivity, Geophys. J Int., , 171, 695-717.
Dziewonski, A. M. \& Anderson, D. L., 1981. Preliminary reference earth model, Phys. Earth Planet. Inter., , 25(4), 297-356.

Dziewoński, A. M., Hager, B. H., \& O’Connell, R. J., 1977. Large-scale heterogeneities in the lower mantle, J. geophys. Res., , 82(2), 239-255.

Faccioli, E., Maggio, F., Quarteroni, A., \& Taghan, A., 1996. Spectraldomain decomposition methods for the solution of acoustic and elastic wave equations, Geophysics, , 61(4), 1160-1174.

Faccioli, E., Maggio, F., Paolucci, R., \& Quarteroni, A., 1997. 2d and $3 \mathrm{~d}$ elastic wave propagation by a pseudo-spectral domain decomposition method, J. Seismology, , 1(3), 237-251.

Fichtner, A., 2010. Full Seismic Waveform Modelling and Inversion., Springer, Heidelberg.

Fichtner, A. \& Hanasoge, S. M., 2017. Discrete wave equation upscaling, Geophys. J Int., , 209(1), 353-357.

Fichtner, A., Bunge, H.-P., \& Igel, H., 2006. The adjoint method in seismology: I. theory, Phys. Earth Planet. Inter., , 157(1-2), 86-104.

Fichtner, A., Kennett, B. L. N., Igel, H., \& Bunge, H.-P., 2009. Full seismic waveform tomography for upper-mantle structure in the Australasian region using adjoint methods., Geophys. J Int., , 179, 1703-1725.

Fichtner, A., van Herwaarden, D., Afanasiev, M., Simute, S., Krischer, L., Cubuk-Sabuncu, Y., Taymaz, T., Colli, L., Saygin, E., Villasenor, A., Trampert, J., Cupillard, P., Bunge, H.-P., \& Igel, H., 2018. The Collaborative Seismic Earth Model: Generation I, Geophys. Res. Lett., , 45, 4007-4016.

French, S. \& Romanowicz, B., 2014. Whole-mantle radially anisotropic shear velocity structure from spectral-element waveform tomography, Geophys. J Int., , 199(3), 1303-1327.

Gazdag, J., 1981. Modeling of the acoustic wave equation with transform methods, Geophysics, , 46(6), 854-859.

Gokhberg, A. \& Fichtner, A., 2016. Full-waveform inversion on heterogeneous HPC systems, Computers and Geosciences, 89, 260-268.

Hunter, J. D., 2007. Matplotlib: A 2d graphics environment, Computing in science \& engineering, 9(3), 90.

Igel, H., 2016. Computational seismology: A practical introduction, Oxford University Press.

Igel, H. \& Gudmundsson, O., 1997. Frequency-dependent effects on travel times and waveforms of long-period S and SS waves, Phys. Earth Planet. Inter., , 104, 229-246.

Igel, H., Djikpesse, H., \& Tarantola, A., 1996. Waveform inversion of marine reflection seismograms for P impedance and Poisson's ratio., Geophys. J Int., , 124, 363-371.

Kluyver, T., Ragan-Kelley, B., Pérez, F., Granger, B. E., Bussonnier, M., Frederic, J., Kelley, K., Hamrick, J. B., Grout, J., Corlay, S., et al., 2016. Jupyter notebooks-a publishing format for reproducible computational workflows., in ELPUB, pp. 87-90.

Komatitsch, D. \& Tromp, J., 2002. Spectral-element simulations of global seismic wave propagation, part II: 3-D models, oceans, rotation, and gravity, Geophys. J Int., , 150, 303-318.

Komatitsch, D., Erlebacher, G., Göddeke, D., \& Michéa, D., 2010. Highorder finite-element seismic wave propagation modeling with mpi on a large gpu cluster, J. Comp. Phys., 229(20), 7692-7714.

Kosloff, D., Reshef, M., \& Loewenthal, D., 1984. Elastic wave calculations by the fourier method, Bull. seism. Soc. Am., , 74(3), 875-891.

Krischer, L., Smith, J., Lei, W., Lefebvre, M., Ruan, Y., de Andrade, E. S., Podhorszki, N., Bozdağ, E., \& Tromp, J., 2016. An adaptable seismic data format, Geophysical Supplements to the Monthly Notices of the Royal Astronomical Society, 207(2), 1003-1011.

Krischer, L., Fichtner, A., Boehm, C., \& Igel, H., 2018. Automated largescale full seismic waveform inversion for north america and the north atlantic, J. geophys. Res., , 123(7), 5902-5928.

Lailly, P. \& Bednar, J., 1983. The seismic inverse problem as a sequence of before stack migrations, in Conference on inverse scattering: theory and application, pp. 206-220, Siam Philadelphia, PA.

Leng, K., Nissen-Meyer, T., \& van Driel, M., 2016. Efficient global wave propagation adapted to 3 -d structural complexity: a pseudospectral/spectral-element approach, Geophysical Supplements to the Monthly Notices of the Royal Astronomical Society, 207(3), 17001721. 
Leng, K., Nissen-Meyer, T., Van Driel, M., Hosseini, K., \& Al-Attar, D., 2019. Axisem3d: broadband seismic wavefields in 3-d global earth models with undulating discontinuities, Geophys. J Int., .

Liu, D. C. \& Nocedal, J., 1989. On the limited memory bfgs method for large scale optimization, Mathematical programming, 45(1-3), 503-528.

Meschede, M. \& Romanowicz, B., 2015. Non-stationary spherical random media and their effect on long-period mantle waves, Geophys. J Int., , 203, 1605-1625.

Nissen-Meyer, T., Dahlen, F., \& Fournier, A., 2007. Spherical-earth fréchet sensitivity kernels, Geophys. J Int., , 168(3), 1051-1066.

Nissen-Meyer, T., Fournier, A., \& Dahlen, F., 2008. A 2-d spectralelement method for computing spherical-earth seismogramsii. waves in solid-fluid media, Geophys. J Int., , 174(3), 873-888.

Nissen-Meyer, T., Driel, M. v., Stähler, S., Hosseini, K., Hempel, S., Auer, L., Colombi, A., \& Fournier, A., 2014. Axisem: broadband 3-d seismic wavefields in axisymmetric media, J. geophys. Res., , (1), 425-445.

Nocedal, J. \& Wright, S., 2006. Numerical optimization, Springer Science $\&$ Business Media.

Oliphant, T. E., 2006. A guide to NumPy, vol. 1, Trelgol Publishing USA.

Patera, A., 1984. A spectral element method for fluid dynamics: laminar flow in a channel expansion, Journal of computational Physics, 54(3), 468-488.

Peter, D., Tape, C., Boschi, L., \& Woodhouse, J. H., 2007. Surface wave tomography: global membrane waves and adjoint methods, Geophys. $J$ Int., , 171, 1098-1117.

Peter, D., Boschi, L., \& Woodhouse, J. H., 2009. Tomographic resolution of ray and finite-frequency methods: a membrane-wave investigation, Geophys. J Int., , 177, 624-638.

Plessix, R.-E., 2006. A review of the adjoint-state method for computing the gradient of a functional with geophysical applications, Geophys. $J$ Int., , 167, 495-503.

Pratt, R. G., Huang, L., Duric, N., \& Littrup, P., 2007. Sound-speed and attenuation imaging of breast tissue using waveform tomography of transmission ultrasound data, in Medical Imaging 2007: Physics of Medical Imaging, vol. 6510, p. 65104S, International Society for Optics and Photonics.

Rietmann, M., Messmer, P., Nissen-Meyer, T., Peter, D., Basini, P., Komatitsch, D., Schenk, O., Tromp, J., Boschi, L., \& Giardini, D., 2012. Forward and adjoint simulations of seismic wave propagation on emerging large-scale gpu architectures, in Proceedings of the International Conference on High Performance Computing, Networking, Storage and Analysis, p. 38, IEEE Computer Society Press.

Rietmann, M., Peter, D., Schenk, O., Uçar, B., \& Grote, M., 2015. Loadbalanced local time stepping for large-scale wave propagation, in Parallel and Distributed Processing Symposium (IPDPS), 2015 IEEE International, pp. 925-935, IEEE.

Ronchi, C., Iacono, R., \& Paolucci, P. S., 1996. The cubed sphere: a new method for the solution of partial differential equations in spherical geometry, J. Comp. Phys., 124(1), 93-114.

Seriani, G. \& Priolo, E., 1994. Spectral element method for acoustic wave simulation in heterogeneous media, Finite Elements in Analysis and Design, 16(3-4), 337-348.

Simute, S., Steptoe, H., Cobden, L., Gokhberg, A., \& Fichtner, A., 2016. Full-waveform inversion of the japanese islands region, J. geophys. Res., , 121(5), 3722-3741.

Sirgue, L., Barkved, O. I., Dellinger, J., Etgen, J., Albertin, U., \& Kommedal, J. H., 2010. Full-waveform inversion: the next leap forward in imaging at Valhall, First Break, 28, 65-70.

Tape, C., Liu, Q., Maggi, A., \& Tromp, J., 2009. Adjoint tomography of the southern california crust, Science, 325(5943), 988-992.

Tarantola, A., 1984. Inversion of seismic reflection data in the acoustic approximation, Geophysics, , 49, 1259-1266.

Tarantola, A., 1988. Theoretical background for the inversion of seismic waveforms, including elasticity and attenuation, in Scattering and Attenuations of Seismic Waves, Part I, pp. 365-399, Springer.

Tromp, J., Tape, C., \& Liu, Q., 2005. Seismic tomography, adjoint methods, time reversal and banana-doughnut kernels, Geophys. J Int., , 160(1), 195-216. van Driel, M., Boehm, C., Krischer, L., \& Afanasiev, M., 2019. Accelerating numerical wave propagation by wavefield-adapted meshes, part i: Forward and adjoint modelling.

Virieux, J. \& Operto, S., 2009. An overview of full-waveform inversion in exploration geophysics, Geophysics, , 74(6), WCC1-WCC26.

Warner, M., Ratcliffe, A., Nangoo, T., Morgan, J., Umpleby, A., Shah, N., Vinje, V., Stekl, I., Guasch, L., Win, C., Conroy, G., \& Betrand, A., 2013. Anisotropic 3d full-waveform inversion, Geophysics, , 78, R59-R80.

Zhu, H., Bozdağ, E., Peter, D., \& Tromp, J., 2012. Structure of the European upper mantle revealed by adjoint tomography, Nat. Geosc., 5, 493-498. 